\title{
Long non-coding RNA EBLN3P promotes the recovery of the function of impaired spiral ganglion neurons by competitively binding to miR-204-5p and regulating TMPRSS3 expression
}

\author{
WENQI JIANG* , ANQUAN PENG* ${ }^{*}$ YICHAO CHEN, BO PANG and ZHIWEN ZHANG \\ Department of Otolaryngology, Head and Neck Surgery, The Second Xiangya Hospital, \\ Central South University, Changsha, Hunan 410011, P.R. China
}

Received September 22, 2019; Accepted February 19, 2020

DOI: $10.3892 / \mathrm{ijmm} .2020 .4545$

\begin{abstract}
Sensorineural hearing loss (SNHL) is one of the major leading causes of hearing impairment, and is typically characterized by the degeneration of spiral ganglion neurons (SGNs). In previous studies by the authors, it was demonstrated that microRNA (miRNA or miR)-204-5p decreased the viability of SGNs by inhibiting the expression of transmembrane protease, serine 3 (TMPRSS3), which was closely associated with the development of SGNs. However, the upstream regulatory mechanism of miR-204-5p was not fully elucidated. The present study found that an important upstream regulatory factor of miR-204-5p, long non-coding RNA (lncRNA) EBLN3P, was expressed at low levels in impaired SGNs, whereas it was expressed at high levels in normal SGNs. Mechanistic analyses demonstrated that lncRNA EBLN3P functioned as a competing endogenous RNA (ceRNA) when regulating miR-204-5p in normal SGNs. In addition, IncRNA EBLN3P regulated TMPRSS3 expression via the regulation of miR-204-5p in normal SGNs. In vitro functional analysis revealed that IncRNA EBLN3P promoted the recovery of the viability of normal SGNs and inhibited the apoptosis of normal SGNs. Finally, the results revealed a recovery-promoting effect of lncRNA EBLN3P on the structure and function of impaired SGNs in models of deafness. On the whole, the findings of the present study demonstrate that lncRNA EBLN3P promotes the recovery of the function of impaired SGNs by competitively binding to miR-204-5p and regulating TMPRSS3 expression. This suggests that lncRNA EBLN3P may be a potential therapeutic target for diseases involving SNHL.
\end{abstract}

Correspondence to: $\operatorname{Dr}$ Zhiwen Zhang, Department of Otolaryngology, Head and Neck Surgery, The Second Xiangya Hospital, Central South University, 139 Middle Renmin Road, Changsha, Hunan 410011, P.R. China

E-mail: zhangzhiwen8@sina.com

*Contributed equally

Key words: lncRNA-EBLN3P, spiral ganglion neurons, sensorineural hearing loss, miR-204-5p, transmembrane protease, serine 3

\section{Introduction}

Sensorineural hearing loss (SNHL) is one of the major leading causes of hearing impairment, and is typically characterized by the degeneration of spiral ganglion neurons (SGNs) (1). Multiple internal and external causative factors greatly affect the development of this disease. Currently, the clinical treatment for severe cases of SNHL is a cochlear implant to locate an electrode array in the scala tympani and electrically stimulate SGNs (2). However, the therapeutic efficacy is reduced due to the continuous degeneration of SGNs (3-5) and a gradual reduction in SGN numbers. Therefore, the urgent investigation of the mechanisms responsible for SGN degeneration and the development of more effective treatment methods are required.

The development of SNHL is influenced by multiple hereditary and developmental factors (6). Numerous genetic loci have been reported to increase the possibility of permanent SNHL caused by ototoxic medications or noise trauma (7). The transmembrane protease, serine 3 (TMPRSS3) gene encodes a proteolytic enzyme that belongs to the subfamily of type II transmembrane serine proteases $(8,9)$. TMPRSS3 is expressed in SGNs, inner hair cells and the stria vascularis of the cochlear duct (10). The TMPRSS3 gene plays crucial roles in the morphological and functional maturation of the inner ear, as well as in maintaining the contents of the endolymph and perilymph $(11,12)$. However, the function of the TMPRSS3 gene in the auditory system has not yet been fully elucidated. A previous study by the authors reported a high TMPRSS3 expression in SGNs of mouse cochleae (13). In addition, it has been demonstrated that microRNA (miRNA or miR)-204-5p suppresses the survival of cochlear SGNs in vitro by directly targeting the TMPRSS3 gene (14). However, the upstream regulatory mechanisms of miR-204-5p have not yet been fully elucidated.

Long non-coding RNAs (lncRNAs) are a type of RNA molecules that are unable to encode proteins, with a length of $>200$ nucleotides (nts) (15). lncRNAs have been defined as RNA molecules functioning as either primary or spliced transcripts, which do not match with the known small RNAs or some structural RNAs (16). IncRNAs have been reported to regulate gene expression through multiple mechanisms, including transcription, post-transcription and chromatin 
modification $(16,17)$. IncRNAs can regulate gene transcription by co-activating transcription factors, interacting with RNA binding proteins (RBPs) or repressing the promoters of targeted genes (18-20). The present study demonstrated that lncRNA EBLN3P promotes the recovery of the function of impaired SGNs by competitively binding to miR-204-5p and regulating TMPRSS3 expression.

\section{Materials and methods}

Establishment of mouse model with kanamycin sulfate-induced hearing impairment. Specific pathogen-free mice (male, 2 months old, weighing 25-35 g), were purchased from the Department of Experimental Animal Science of Central South University. Their auditory brainstem response (ABR) threshold was normal, and middle and inner ear diseases were excluded. The mice were randomly divided into 2 groups as follows: An experimental group and a control group. The mice in the experimental group were intramuscularly injected with 500 mg/kg/day kanamycin sulfate (040810OG; Ameresco, Inc.) for 14 days, while those in the control group were injected with an equal volume of normal saline for 14 days, as previously described (21). For each group at the beginning, 8 mice in total were used for the whole experimental procedure. All the mice were supplied with water and food ad libitum and allowed to acclimatize to their environment for a period of 7 days prior to being used in the corresponding experiment. Humane endpoints were applied for all mice in the experiments. The care of the laboratory animals and animal experimentation were performed in accordance with animal ethics guidelines and approved by the Animal Care and Use Committee at Xiangya School of Medicine from Central South University. Humane endpoints were used and mice displaying severe illness were euthanized prior to the end of the experiments to minimize suffering and distress. The health and behavior of the mice were monitored twice per day. The criteria for determining when the animals should be euthanized included weight loss, loss of appetite, lack of feeding or drinking, weakness, ruffled fur, signs of severe organ system dysfunction and not responsive to treatment. The duration of the experiment was approximately 14 days. In the process of the experiment, one mouse from the experimental group was found to have sustained weight loss (18\% weight loss was observed) and also exhibited a loss of appetite; this this mouse was euthanized by $\mathrm{CO}_{2}$ exposure according to the IACUC policy. The optimal flow rate was set $20 \%$ of the chamber volume per minute until the mouse became unconscious. At the completion of all the in vivo experiments, 6 mice from each group were used for quantification, and the remaining 3 mice from the 2 groups were euthanized by $\mathrm{CO}_{2}$ exposure as described above. The death of the mice was verified by monitoring cardiac cessation and respiratory arrest. All the best efforts were made to minimize the suffering and distress of the animals.

$A B R$ test. The 6 mice from each group mentioned above, which were used for quantification were also used for the ABR test. Anesthesia was performed by an intraperitoneal injection of $2 \%$ sodium pentobarbital $(40 \mathrm{mg} / \mathrm{kg}$, prepared with physiological saline), as previously described (22). After 5-10 min, the stinging and blink reflexes of the mice disappeared, and the breathing slowed down, thereby indicating that the state of anesthesia was moderate and the subsequent experiment could be initiated (23). The moderately anesthetized mice were fixed on an experimental bench in prone position, and their body temperature was maintained at a constant level during the experiment. The placement of three electrodes to detect ABR was carried out as follows: The recording electrode was placed subcutaneously in the middle of the cranial crest; the referred electrode was placed under the skin of the mastoid of the ear to be measured; and the ground wire was placed under the skin of the papillary part of the contralateral ear. The experimental mice, the electrodes, the front signal amplifier of the ABR detection instrument and the sound stimulator were placed in the acoustic- and electric-shielded room, while the other instruments were placed outside the shielded room.

The Smart-EP function option of the SmartEP3.91USBez software (Intelligent Hearing Systems) stimulates the tone-pip stimuli generated by the high-frequency sound transducer, and is sent to the external auditory canal of the experimental animal by $0.5 \mathrm{~cm}$ through a plastic tube of $\sim 2-\mathrm{mm}$ diameter. The output signal is input to the computer via a bio-signal pre-amplifier and is monitored by a computer monitor. A tone-pip of $8 \mathrm{kHz}$ frequency was selected as the stimulation sound; the stimulation rate was 39.10 counts/sec; the stimulation duration was 5 cycles; and the rise and fall times were 2 cycles. A trapezoidal envelope was used; the recording analysis time was $16.0 \mathrm{msec}$, and the number of superpositions was 1,024. Tone-pip starts from $90 \mathrm{~dB}$ of sound pressure level (dBSPL) and is first decremented by $10 \mathrm{dBSPL}$ and then by $5 \mathrm{dBSPL}$. The minimum stimulation intensity that was able to distinguish I or II wave patterns was determined as a threshold, and was repeated twice.

Isolation and in vitro culture of SGNs. The protocol for SGN isolation and culture was based on a previous study (24). As the previously described protocol (24) was performed on rats, and the experiment in the present study was performed on mice, necessary modifications were therefore made according to the similar anatomic structures of the Corti organ, but different sizes of the corresponding organs. A total of 60 specific pathogen-free mice (male, 2 months old, weighing 25-35 g) were purchased from the Department of Experimental Animal Science of Central South University. Half of the mice were used directly for SGN isolation, while the remaining mice were used for the establishment of the deafness models by kanamycin sulfate-induced hearing impairment as described above, followed by SGN isolation. SGNs were dissected from the cochleae of all the mice as previously described (25). In detail, SGNs were isolated from the modiolus, followed by the removal of the sensory epithelium from the organ of Corti. The SGNs were then treated with $0.25 \%$ trypsin for $15 \mathrm{~min}$ at $37^{\circ} \mathrm{C}$, followed by trypsin neutralization with $10 \%$ fetal bovine serum (FBS) in Dulbecco's modified Eagle's medium (DMEM). Subsequently, the SGNs were collected by centrifugation at $4^{\circ} \mathrm{C}, 500 \mathrm{x}$ for $7 \mathrm{~min}$, and triturated to form a single-cell suspension in DMEM/F12 supplemented with N2 (primary rat embryonic hippocampal neurons, tumor cell lines of neuronal origin) and B27 (primary rat embryonic hippocampal neurons, primary rat neurons from the striatum, substantia nigra, septum) cells. Morphological observation of the SGNs was performed using a DMI3000B microscope 
(Leica, Ernst-Leitz). The in vivo experimental protocol was approved by the Animal Care and Use Committee at Xiangya School of Medicine from Central South University. Euthanasia was performed by an intraperitoneal injection of sodium pentobarbital $(100 \mathrm{mg} / \mathrm{kg}$, prepared with physiological saline) (26), and the means used to verify mouse death were as follows: No spontaneous breathing for a continuous duration of 2-3 min, and no blinking reflex.

miRNA target predictions. Predicted binding targets of miR-204-5p were analyzed by searching online databases, such as mirTarget2 (http://mirdb.org/), microRNA.org (www. microrna.org) and starBase v2.0 (starbase.sysu.edu.cn/starbase2).

Plasmid construction and cell transfection. Human EBLN3P genes were amplified by reverse transcription-PCR (RT-PCR). In detail, the total RNAs were extracted from the SGNs cells using TRIzol (Invitrogen; Thermo Fisher Scientific, Inc.). The RNA concentrations were determined by a NanoDrop ND-1000 instrument (NanoDrop Technologies; Thermo Fisher Scientific, Inc.). cDNA was produced by reverse transcription using the PrimeScript $^{\mathrm{TM}}$ One Step RT-PCR kit Ver.2 according to the manufacturer's instructions (Takara). PCR amplification was performed using the following primers: IncRNA EBLN3P sense, 5'-GTGTTGTCCCGGAAGTGCCTTCTC-3' and antisense, 5'-TTGAAGGTTTGCCTTCTCTGAATAG-3'. The parameters for PCR amplification were as follows: $5 \mathrm{~min}$ at $95^{\circ} \mathrm{C}$, followed by 35 cycles of $60 \mathrm{sec}$ at $95^{\circ} \mathrm{C}, 60 \mathrm{sec}$ at $58^{\circ} \mathrm{C}$ and $90 \mathrm{sec}$ at $72^{\circ} \mathrm{C}, 8 \mathrm{~min}$ at $72^{\circ} \mathrm{C}$. The PCR amplification were performed on an Eppendorf Mastercycler X50h instrument (Eppendorf). The EBLN3P gene was then subcloned into the expression vector, pcDNA3.1 (Invitrogen; Thermo Fisher Scientific), named pcDNA3.1-EBLN3P. The 1,200-nt gene fragment at the 5' end of either EBLN3P containing the two predicted binding sites (regions '314-322' and '973-981' in the 1,200-nt EBLN3P fragment) for miR-204-5p or EBLN3P-mutant (mut) (with point mutations in the miR-204-5p response elements) was amplified using a common PCR method, and then subcloned into the pmirGLO vector (Promega Corp.) for subsequent luciferase reporter analyses. The plasmids pcDNA3.1-MS2, pcDNA3.1-MS2-EBLN3P and pcDNA3.1-MS2-EBLN3P-mut (miR-204-5p) were constructed for the following RNA immunoprecipitation (RIP) assay.

The double-stranded miR-204-5p mimics, negative control miRNAs, miR-204-5p inhibitors and recombinant adenoviruses containing the overexpression vector of EBLN3P or the control vector were produced by GeneChem, Inc. The recombinant plasmids pLV4-small hairpin RNA (shRNA)/EBLN3P and pLV4-shRNA/argonaute 2 (AGO2), with their respective non-targeting control pLV4-shRNA-negative control (NC), were constructed. All transient transfections were performed using Lipofectamine 3000 reagent (Invitrogen; Thermo Fisher Scientific, Inc.) in accordance with the manufacturer's instructions. To improve the transfection efficiency in neuronal cells, the positively transfected cells were subsequently screened by flow cytometry following transfection with a GFP-labeled for $48 \mathrm{~h}$, and the follow-up experiment then commences. The transfection efficiency was evaluated by fluorescence microscopy observation.
Reverse transcription-quantitative PCR (RT-qPCR). For TMPRSS3 gene quantification, and the determination of lncRNA EBLN3P and miR-204-5p expression, total RNA, which contained all miRNAs and lncRNAs, was extracted using TRIzol (Invitrogen; Thermo Fisher Scientific, Inc.). RNA concentrations were measured with a NanoDrop ND-1000 instrument (NanoDrop Technologies; Thermo Fisher Scientific, Inc.), and the final samples were stored at $-80^{\circ} \mathrm{C}$. cDNA was produced by reverse transcription using the SuperScript ${ }^{\circledR}$ III RT-PCR kit according to the manufacturer's instructions (Thermo Fisher Scientific, Inc.). RT-qPCR was performed using the following primers: TMPRSS3 sense, 5'-AGTGGGGTAGACGGAGACCT-3' and antisense, 5'-CACTGAACCCTTCCTGGTTT-3'; lncRNA EBLN3P sense, 5'-TACGCGTTTTGGTCCCTGTT-3' and antisense, 5'-GCCACTTGGCTCAAAAGACTG-3'; miR-204-5p sense, 5'-ACACTCCAGCTGGGTTCCCTTTGTCAT-3' and antisense, 5'-CTCAACTGGTGTCGTGGAGTCGGCAATTCA GTTGAGAGGCATGG-3'; $\beta$-actin sense, 5'-AGCAGCATC GCCCCAAAGTT-3' and antisense, 5'-GGGCACGAAGGC TCATCATT-3'; and U6 sense, 5'-CTCGCTTCGGCAGC ACA-3' and antisense, 5'-AACGCTTCACGAATTTGCGT-3'. The parameters for PCR quantification were as follows: 2 min at $95^{\circ} \mathrm{C}$, followed by 40 cycles of $15 \mathrm{sec}$ at $95^{\circ} \mathrm{C}$ and $30 \mathrm{sec}$ at $60^{\circ} \mathrm{C}$. The results of qPCR were defined using the quantification cycle $(\mathrm{Cq})$, and $2^{-\Delta \Delta \mathrm{Cq}}$ was used to calculate the relative expression levels (27). All the PCR assays were performed on an ABI 7500 instrument (Applied Biosystems; Thermo Fisher Scientific, Inc.).

RIP assay. SGNs were co-transfected with pMS2-GFP and pcDNA3.1-MS2, or pMS2-GFP and pcDNA3.1-MS2-EBLN3P, or pMS2-GFP and pcDNA3.1-MS2-EBLN3P-mut (miR-204-5p). After $48 \mathrm{~h}$, the above-mentioned SGNs were subjected to RIP assay using an anti-GFP antibody (dilution: 1:100, cat. no. 11814460001; Roche Diagnostics) and the Magna RIP ${ }^{\mathrm{TM}}$ RNA-Binding Protein Immunoprecipitation kit (EMD Millipore) in accordance with the manufacturer's instructions.

RNA pull-down assay. IncRNA EBLN3P or lncRNA-EBLN3P-mut (miR-204-5p) were in vitro transcribed from the vector pSPT19-EBLN3P or pSPT19-EBLN3P-mut (miR-204-5p), respectively, and biotin-labeled with Biotin RNA Labeling mix and T7 RNA polymerase (Roche Diagnostics), followed by treatment with RNase-free DNase I and purificatiob with RNeasy Mini kit (Qiagen $\mathrm{GmbH}$ ). Cell lysates from SGNs $(1 \mu \mathrm{g})$ were co-incubated with $3 \mu \mathrm{g}$ biotinylated transcripts for $1 \mathrm{~h}$ at $25^{\circ} \mathrm{C}$, and then the co-incubated complexes were separated with streptavidin agarose beads (Invitrogen; Thermo Fisher Scientific, Inc.), followed by RT-qPCR analysis.

Luciferase reporter assay. 293T cells (ATCC, $~ 8,000$ cells per well) or primary isolated SGNs (12,000 cells per well) were seeded into 96 -well plates, and co-transfected with $50 \mathrm{nmol} / 1$ miR-204-5p mimic (or NC), 50 ng luciferase reporter plasmid and 5 ng pRLCMV Renilla luciferase reporter plasmid using Lipofectamine 3000 reagent (Invitrogen; Thermo Fisher Scientific, Inc.). Following $48 \mathrm{~h}$ of incubation, the Firefly and Renilla luciferase activities were measured using a 
Dual-Luciferase ${ }^{\circledR}$ Reporter Assay kit (Promega Corp.). Data are presented as the relative ratio of Firefly luciferase activities to Renilla luciferase activities.

Western blot analysis. SGNs subjected to the different treatments were firstly washed twice with PBS solution, and then lysed in RIPA lysis buffer (Beyotime Institute of Biotechnology). The concentration of the protein samples was determined by the BCA method. A total of $40 \mu \mathrm{g}$ protein for each sample was then loaded and separated by $10 \%$ SDS/PAGE, followed by transfer onto PVDF membranes (EMD Millipore). The membranes with targeted proteins were blocked with $5 \% \mathrm{BSA}$ at $37^{\circ} \mathrm{C}$ for $2 \mathrm{~h}$, and then incubated with primary antibodies overnight at $4^{\circ} \mathrm{C}$. The membranes were then washed 3 times, and incubated at $37^{\circ} \mathrm{C}$ for $1 \mathrm{~h}$ with an HRP-conjugated anti-rabbit secondary antibody (1:5,000, ab97051, Abcam), followed by the detection of the immunoreactive protein bands with an Odyssey Scanning system. The anti-AGO2 rabbit monoclonal antibody $(\mathrm{mAb})(\# 2897$, $1: 1,000)$ and anti-cleaved caspase-3 rabbit mAb (\#9664, 1:1,000) were purchased from Cell Signaling Technology, Inc., while the anti-TMPRSS3 rabbit polyclonal antibody (\#PA5-35325, 1:1,000) was purchased from Invitrogen (Thermo Fisher Scientific, Inc.). Anti- $\beta$-actin rabbit monoclonal antibody (1:5,000, ab179467, Abcam) was used as the internal control. Densitometric analysis was performed using the Scion Imaging application (4.0, Scion Corp.).

Cell viability assay. The SGN suspension $\left(5 \times 10^{4}\right.$ cells $\left./ \mathrm{ml}\right)$ was seeded into a 96-well plate and incubated overnight at $37^{\circ} \mathrm{C}$. After being subjected to the different treatments for $48 \mathrm{~h}$, the SGNs were incubated with MTT solution for $1 \mathrm{~h}$. Subsequently, $100 \%$ DMSO were added to each well, and the absorbance of each well was measured at $570 \mathrm{~nm}(28,29)$ using a microplate enzyme-linked immunosorbent assay reader (Labsystems Dragon, Wellscan). Cell viability was also measured with Trypan blue staining with the Trypan blue staining kit (Sangon Biotech) at $37^{\circ} \mathrm{C}$ for $3 \mathrm{~min}$. Lactate dehydrogenase $(\mathrm{LDH})$ release was quantified to determine cell death using the CytoTox $96^{\circledR}$ Non-Radioactive Cytotoxicity assay (Promega Corp.) according to manufacturer's instructions and as previously described (30). Values were normalized to protein concentrations to account for differences in cell numbers per well. All the experiments were performed $\geq 3$ times.

Flow cytometry. SGNs were seeded into 6-well plates at a density of $2 \times 10^{5}$ cells/well with DMEM supplemented with $10 \%$ FBS. Subsequently, the cells were harvested by trypsinization and washed twice with cold PBS solution. To measure cell apoptosis, the pre-treated SGNs were fixed in ice-cold $70 \%$ ethanol at $4^{\circ} \mathrm{C}$ for $1 \mathrm{~h}$. After washing once with cold PBS, the SGNs were subsequently stained with propidium iodide (P1304MP, Invitrogen; Thermo Fisher Scientific) and anti-Annexin-V antibody (563973, BD Biosciences) at $4^{\circ} \mathrm{C}$ for $1 \mathrm{~h}$. Finally, the SGNs were analyzed with a fluorescence-activated cell sorting instrument (BD Biosciences).

Adenovirus injection. A capillary with a tip of $\sim 100 \mu \mathrm{m}$ was made with a P-97 puller. After the mice were anesthetized by an intraperitoneal injection of $10 \%$ of chloral hydrate $(300 \mathrm{mg} / \mathrm{kg}$ of mouse body weight, and no mice exhibited signs of peritonitis, pain or discomfort) (31), the round window membrane was pierced with a capillary and fixed in situ. Adenovirus for EBLN3P overexpression $(2 \mu \mathrm{l})$ was injected into the inner ear for 14 days by capillary using a Hamilton microsyringe at an injection rate of $0.1 \mu \mathrm{l} / \mathrm{min}$ to avoid damage to the inner ear tissue (32). Herein, another batch of specific pathogen-free mice (male, 2 months old, weighing 25-35 g) was purchased (total number, 12) from the Department of Experimental Animal Science of Central South University. They were used for the establishment of the deafness models by kanamycin sulfate-induced hearing impairment as described above, followed by adenovirus injection treatment. The 12 mice were divided into 2 groups as follows: 6 mice were treated with control adenovirus, and another 6 mice were treated with adenovirus for EBLN3P overexpression. In each group, 6 mice were used for quantification. Euthanasia was performed as described above.

Hematoxylin and eosin $(H \& E)$ staining and immunohistochemistry (IHC). Paraffin-embedded cochlear SGN tissue samples from mouse cochleae were selected for H\&E staining and IHC. The H\&E staining kit was purchased from Abcam (ab245880). In detail, the sections were deparaffinized if needed and hydrated in distilled water. Hematoxylin [Mayer's (Lillie's Modification)] was then applied to completely cover the tissue sections followed by incubation for $5 \mathrm{~min}$. The slides were then rinsed in two changes of distilled water to remove the excess stain. Bluing reagent was applied to completely cover the tissue sections followed by incubation for $10-15 \mathrm{sec}$. The sections were again rinsed in two changes of distilled water. The slides were then dipped in absolute alcohol and the excess was blotted off. Adequate Eosin Y Solution (Modified Alcoholic) was then applied to completely cover the tissue sections followed by incubation for 2-3 min. The slices were subsequently rinsed using absolute alcohol, followed by dehydration in 3 changes of absolute alcohol. The slides were then cleared and mounted in synthetic resin. IHC for $\beta$-tubulin (1:250, ab179513, Abcam), TMPRSS3 (1:200, ab167160, Abcam) and neurotrophin-3 (NT-3, 1:100, ab216491, Abcam) was carried out using a rabbit monoclonal or polyclonal primary antibody at $4^{\circ} \mathrm{C}$ overnight, an HRP-conjugated goat anti-rabbit IgG secondary antibody $\left(1: 1,000\right.$, ab150077, Abcam) at $37^{\circ} \mathrm{C}$ for $1 \mathrm{~h}$, and DAB staining solution (ab64238, Abcam) at room temperature for $10 \mathrm{~min}$. The staining results were assessed with a DMI3000B microscope (Leica, Ernst-Leitz, Wetzlar, Germany).

Fluorescence in situ hybridization (FISH). Paraffin-embedded cochlear spiral ganglion neuron tissue samples from mouse cochleae were selected for in situ hybridization with FITC-labeled lncRNA EBLN3P DNA probe (ShineGene). The paraffin-embedded tissues were cut into $5-\mu \mathrm{m}$-thick tissue sections for FISH using a Leica RM2235 Manual Rotary Microtome (Leica Microsystems $\mathrm{GmbH}$ ). After dewaxing the slides with xylem $(2 \times 10 \mathrm{~min})$ and methanol $(2 \times 5 \mathrm{~min})$. The slides were exposed to microwaves $(720 \mathrm{~W})$ in $10 \mathrm{mM}$ citric acid monohydrate ( $\mathrm{pH}$ 6.0) for $30 \mathrm{sec}$, followed by immersion in $1 \mathrm{M}$ sodium thiocyanate for $10 \mathrm{~min}$ at $80^{\circ} \mathrm{C}$, and in protease solution for $10 \mathrm{~min}$. The tissue sections were then washed with pure $\mathrm{H}_{2} \mathrm{O}$, air-dried and dehydrated in ascending gradients of alcohol. The lncRNA EBLN3P probe mixtures $(10 \mathrm{ml})$ 



$E$

DAP


ProbeEBLN3P
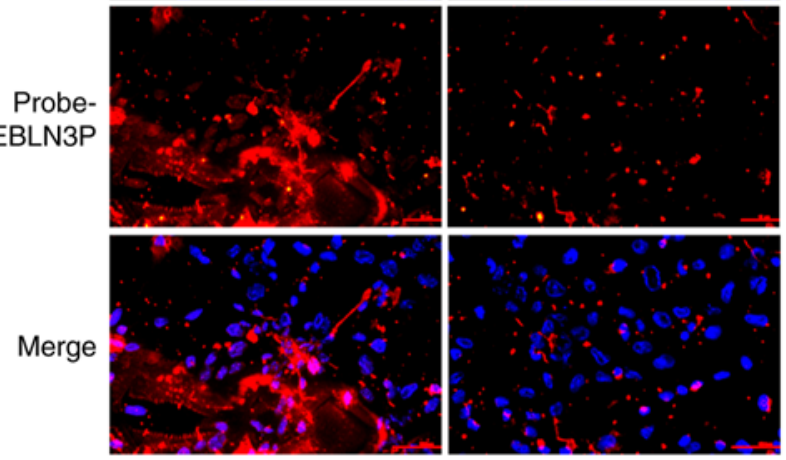

$\mathrm{G}$

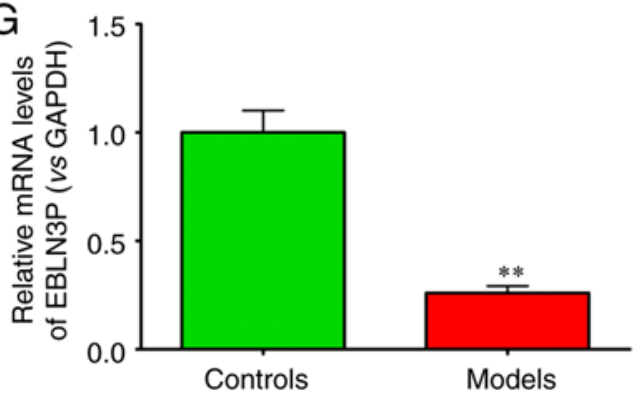

C

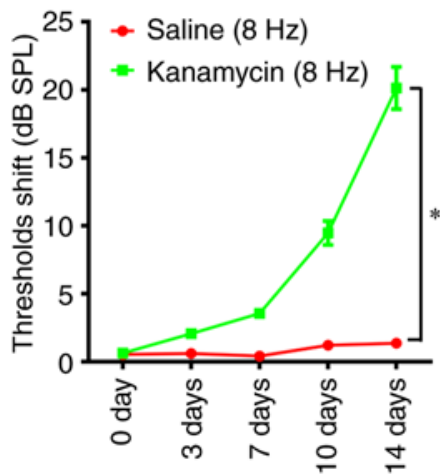

$\mathrm{F}$


Model

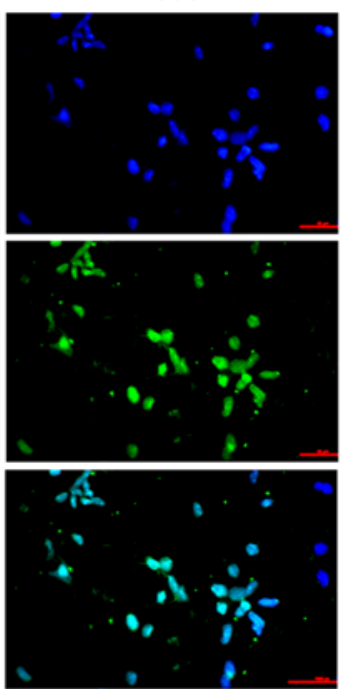

H



Figure 1. Expression of lncRNA EBLN3P and miR-204-5p in models of deafness. (A) Schematic representation of the predicted binding sites of miR-204-5p in the lncRNA EBLN3P transcript according to online prediction software (www.biostars.org). (B) Schematic representation of models of deafness. (C) Estimation of acoustic sensibility in mice. The green and red dots represent the mean of threshold shift to the 8-kHz stimuli before and after the injection of kanamycin and saline at days $0,3,7,10$ and 14 . $^{*} \mathrm{P}<0.05$ (Student's t-test), vs. control saline. (D) SGNs were dissected from P4-P6 mouse cochlea by trypsinization. (E and F) Fluorescence in situ hybridization was performed to detect the expression of lncRNA EBLN3P and miR-204-5p in primary SGNs in a model of deafness. (G and H) RT-qPCR was performed to detect the expression of lncRNA EBLN3P and miR-204-5p in primary SGNs from the mice in the model of deafness. IncRNA, long non-coding RNA; miR, microRNA; SGN, spiral ganglion neuron. ${ }^{* *} \mathrm{P}<0.01$ (Student's t-test), vs. controls.

were added to the air-dried tissue sections. The ThermoBrite System (Abbott Molecular) was used for denaturation and hybridization. Subsequently, the tissue sections were washed with $0.4 \mathrm{X}$ saline sodium citrate solution for $2 \mathrm{~min}$ at $70^{\circ} \mathrm{C}$, and $2 \mathrm{X}$ saline sodium citrate solution for a further $5 \mathrm{~min}$ at room temperature. Finally, $10 \mathrm{ml}$ DAPI (Beyotime Institute of Biotechnology) was added to the tissue sections, followed by fluorescence microscopy (Leica, Ernst-Leitz) evaluation. The final data were evaluated by two investigators.
TUNEL assay. Using a DeadEnd ${ }^{\mathrm{TM}}$ Colorimetric TUNEL System kit (Promega Corp.), TUNEL assay was performed to detect the apoptosis of the SGNs in accordance with the manufacturer's instructions. The observation and capture of digital images were conducted using a Nikon E80i microscope (Nikon Corp.).

Statistical analysis. Data are presented as the means \pm standard deviation from $\geq 3$ independent experiments. A normality 
B


D

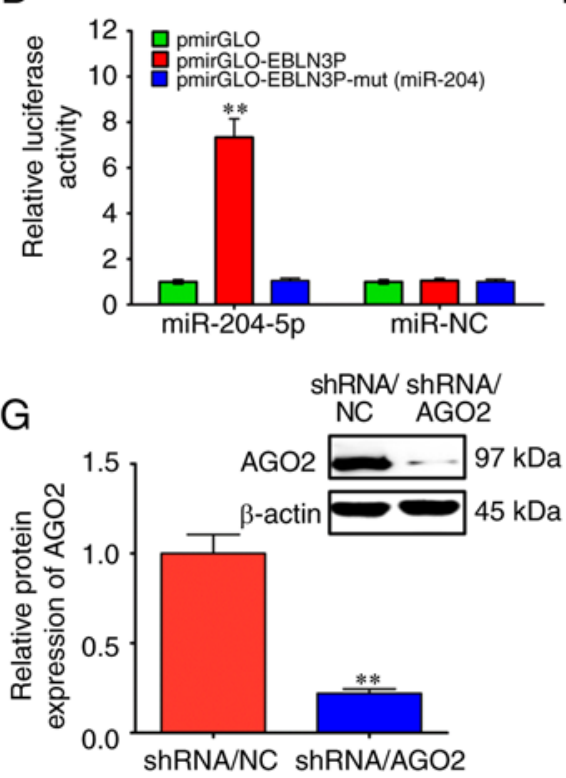

$\mathrm{E}$

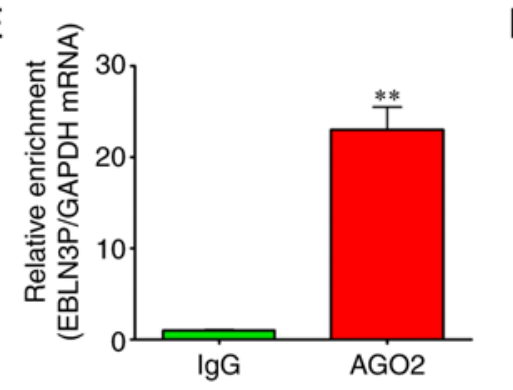

$\mathrm{H}$

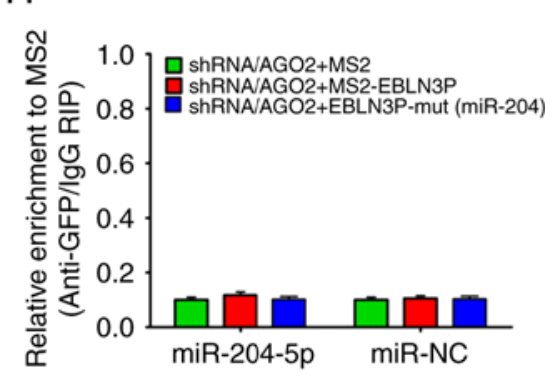

$\mathrm{F}$
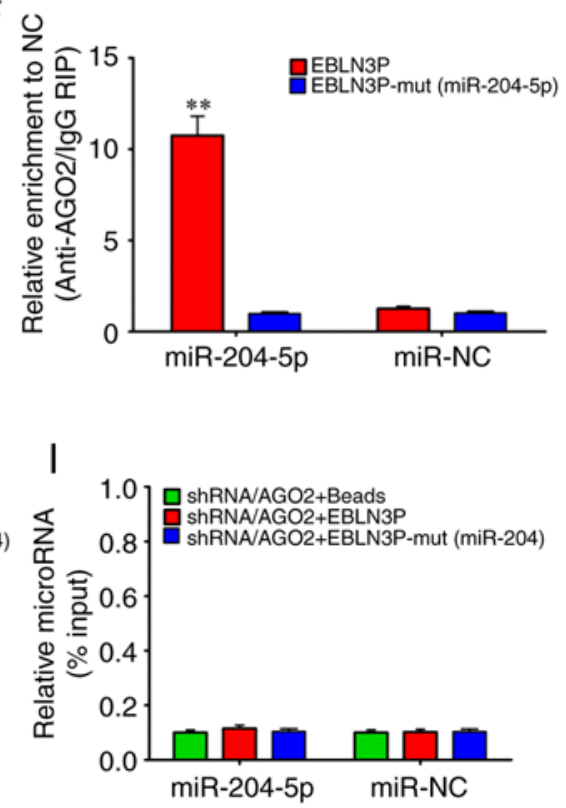

Figure 2. lncRNA EBLN3P functions as a competing endogenous RNA by regulating miR-204-5p in normal SGNs. (A and B) MS2-RIP assay followed by RT-qPCR to detect endogenous miRNAs associated tightly with lncRNA EBLN3P. (C) Cell lysates of normal SGNs were incubated with biotin-labeled EBLN3P. After pull-down, the miRNAs were separated and assayed by RT-qPCR. (D) Luciferase activities in normal SGNs cells co-transfected with miR-204-5p and empty luciferase reporters, IncRNA EBLN3P wild transcript or lncRNA EBLN3P mutant transcript. Data are presented as the relative ratio of Firefly luciferase activity to Renilla luciferase activity. (E) RIP assay of the binding between lncRNA EBLN3P and AGO2 using control IgG and AGO2 special antibody. IncRNA EBLN3P and GAPDH expression was quantified using RT-qPCR, and analyzed as enrichment in RNA-binding protein RIP in contrast to control IgG RIP. (F) Anti-AGO2 RIP was performed in SGNs that were transiently transfected with miR-204-5p mimics, followed by RT-qPCR to detect lncRNA EBLN3P or lncRNA EBLN3P-mut (miR-204-5p) associated with AGO2. (G) After silencing AGO2 in normal SGNs for $48 \mathrm{~h}$, the protein expression level of AGO2 was assessed by western blotting. (H) After silencing the AGO2 gene in normal SGNs for $48 \mathrm{~h}$, MS2-RIP analysis followed by RT-qPCR was performed to detect miR-204-5p endogenously associated with lncRNA EBLN3P. (I) After silencing AGO2 in normal SGNs for $48 \mathrm{~h}$, the cells were lysed, and the cell lysates were incubated with biotin-labeled lncRNA EBLN3P. After pull-down, the whole miRNAs were extracted, and miR-204-5p was measured by RT-qPCR. " P<0.01 (ANOVA with Tukey's post hot-test). lncRNA, long non-coding RNA; miR, microRNA; miRNA, microRNA; SGN, spiral ganglion neuron; RIP, RNA immunoprecipitation; RIP, RNA immunoprecipitation; AGO2, argonaute 2.

test (D'Agostino-Pearson) was used to analyze the distribution of all datasets. A two-tailed Student's t-test and one-way ANOVA were performed to analyze the data using SPSS 20.0 (IBM Corp.). The paired-samples t-test was performed to compare the mean of two matched groups of cases. ANOVA was used for multiple comparisons, and Tukey's post hoc test was performed after ANOVA. $\mathrm{P}<0.05$ was considered to indicate a statistically significant difference.

\section{Results}

Expression of IncRNA EBLN3P and miR-204-5p in models of deafness. By bioinformatics analysis using online databases, such as mirTarget2, microRNA.org and starBase v2.0, it was revealed that IncRNA EBLN3P could effectively bind to miR-204-5p (Fig. 1A). Therefore, models of deafness were established (Fig. 1B). The ARB test indicated that compared with the control saline-treated group, kanamycin significantly increased the threshold (Fig. 1C). Subsequently, mouse primary SGNs were separated (Fig. 1D). By FISH, the expression of IncRNA EBLN3P and miR-204-5p in mouse primary SGNs was detected. The results demonstrated that lncRNA EBLN3P was overexpressed, while miR-204-5p was expressed at low levels in primary SGNs in the control mice. However, in the primary SGNs from the mice in the deafness models, lncRNA EBLN3P was expressed at low levels, while miR-204-5p was overexpressed (Fig. 1E and F). RT-qPCR further confirmed the results of FISH (Fig. 1G and H).

IncRNA EBLN3P functions as a ceRNA, regulating miR-204-5p in normal SGNs. Numerous mRNAs or IncRNAs have been reported to function as ceRNAs by binding to targeted miRNAs 


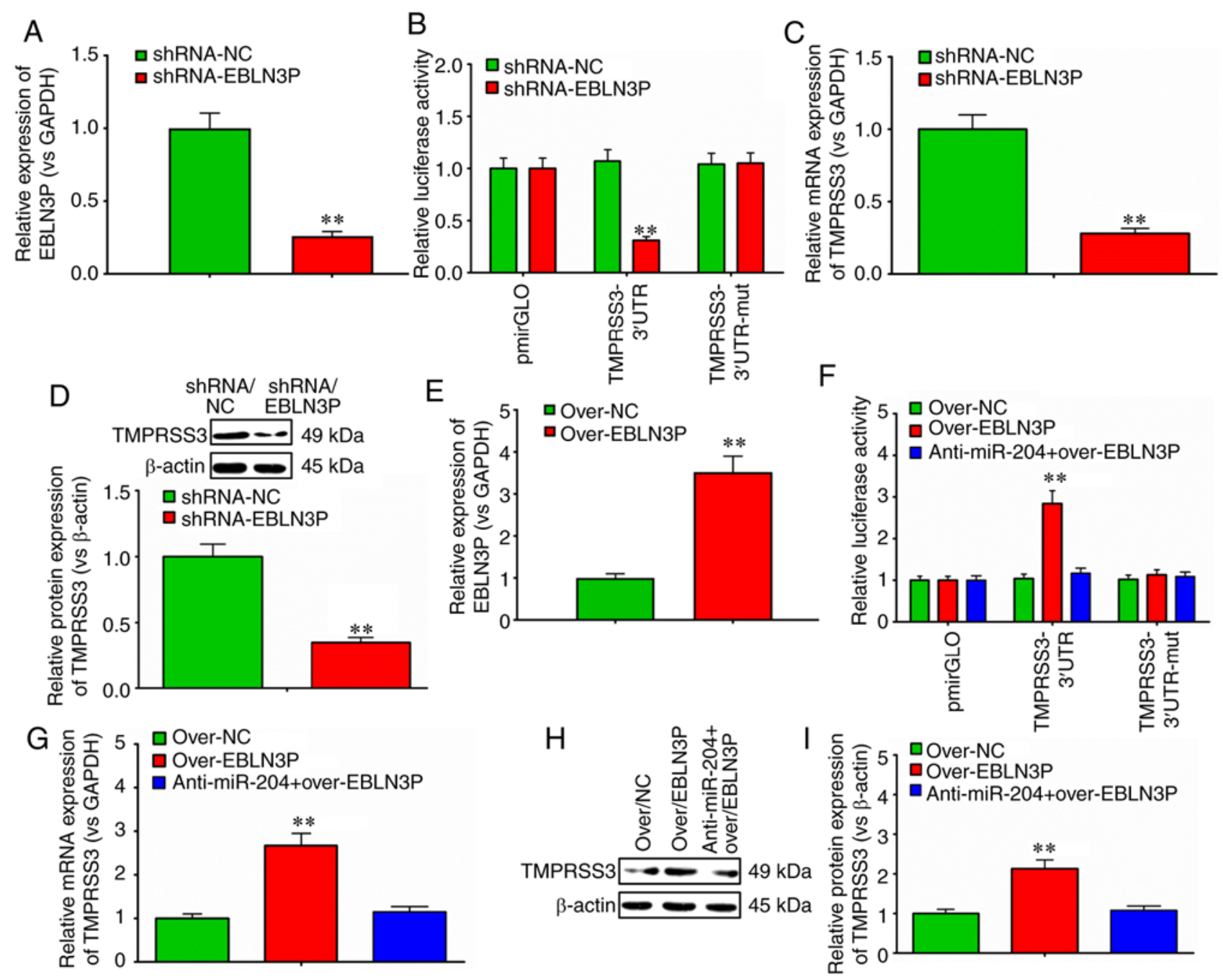

Figure 3. IncRNA EBLN3P regulates the expression of TMPRSS3 through miR-204-5p in normal SGNs. (A) RT-qPCR was performed to detect the effects of lncRNA EBLN3P knockdown on the expression of EBLN3P in primary normal SGNs. (B) Luciferase activity was evaluated to observe the effect of lncRNA EBLN3P knockdown on the psiCHECK-2/TMPRSS3 wild-type 3'-UTR, psiCHECK-2/TMPRSS3 mutated 3'-UTR and empty 3'-UTR vectors (control). (C) RT-qPCR was performed to detect the effects of lncRNA EBLN3P knockdown on the mRNA expression of TMPRSS3 in primary normal SGNs. (D) Western blot analysis was performed to detect the effects of lncRNA EBLN3P knockdown on the protein expression of TMPRSS3 in primary normal SGNs. (E) RT-qPCR was performed to detect the effects of lncRNA EBLN3P overexpression on the expression of EBLN3P in primary normal SGNs. (F) Normal SGNs were treated with over-NC, over-lncRNA EBLN3P or anti-miR-204-5p + over-lncRNA EBLN3P, and the luciferase activity was evaluated. (G-I) Normal SGNs were treated with control over-NC, over-lncRNA EBLN3P or anti-miR-204-5p + over-lncRNA EBLN3P. RT-qPCR and western blot analysis was performed to detect the mRNA and protein expression of TMPRSS3 in primary normal SGNs. ${ }^{* *} \mathrm{P}<0.01$ (Student's t-test) vs. NCs in panels A, B, C, D and E. * P $<0.01$ (ANOVA with Tukey's post hoc test) in panels F, G and I. lncRNA, long non-coding RNA; SGN, spiral ganglion neuron; TMPRSS3, transmembrane protease, serine 3; UTR, untranslated region; NC, negative control; miR, microRNA.

and regulating downstream gene expression. To confirm the direct binding between IncRNA EBLN3P and miR-204-5p, RIP assay was performed to pull down the miRNAs binding to endogenous lncRNA EBLN3P (Fig. 2A). RT-qPCR demonstrated that lncRNA EBLN3P RIP in the SGNs was significantly enriched for miR-204-5p, in contrast to the empty vector (MS2), IgG, non-targeting miRNA (miR-67) and lncRNA EBLN3P with mutations in miR-204-5p targeting sites (Fig. 2B). To further validate the strong interaction between lncRNA EBLN3P and miR-204-5p, lncRNA EBLN3P was transcribed, labeled with biotin and used to successfully pull down miR-204-5p (Fig. 2C). To validate the specific association between lncRNA EBLN3P and miR-204-5p,dual-luciferase reporters (pmirGLO) containing the 5'end 1,200-nt of IncRNA-EBLN3P, either wild-type (WT) or mutated miR-204-5p binding sites were generated. When
miR-204-5p was co-transfected with pmirGLO-EBLN3P, the luciferase activities of pmirGLO-EBLN3P decreased, while the luciferase activities of empty vector pmirGLO and pmirGLO-EBLN3P-mut (miR-204-5p) were not affected (Fig. 2D).

In general, miRNAs bind to the 3'-untranslated region (UTR) of their targeted mRNA to repress the translation of the target mRNA or promote mRNA degradation through an important protein, AGO2 (33). Mature miRNAs can be transported to the nucleus from the cytoplasm in the presence of AGO2 protein, as well as TNRC6A, importin and other proteins (34-36). In the present study, to investigate whether miR-204-5p is regulated by lncRNA EBLN3P via such a mechanism, the strong interaction between lncRNA EBLN3P and AGO2 was further confirmed using an anti-AGO2-specific binding antibody (Fig. 2E). An 
A

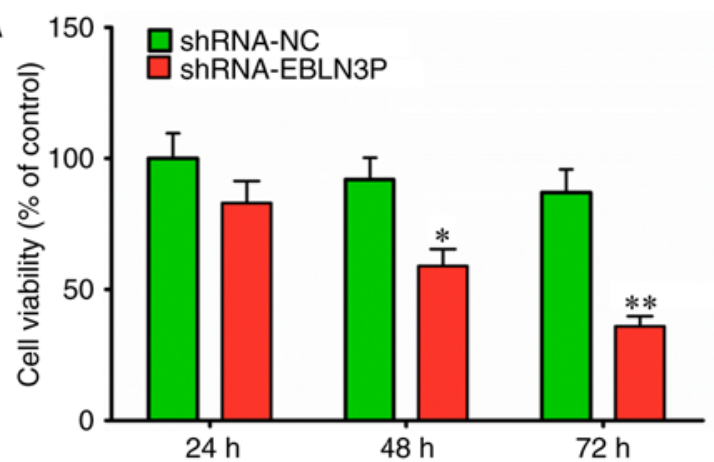

C



E

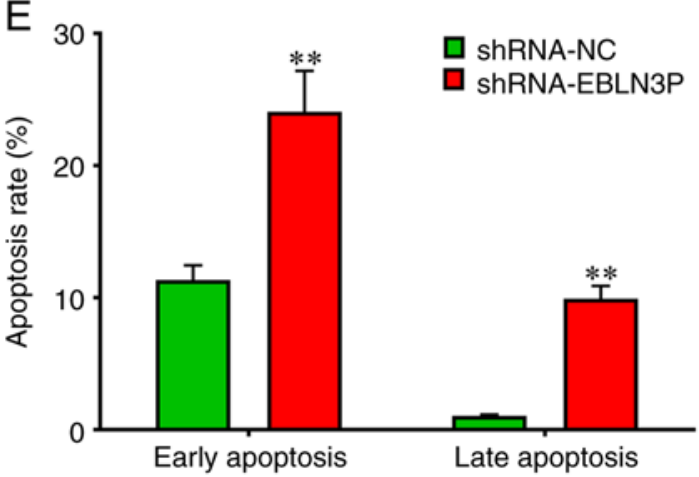

B $\quad 150]$ 口 5 ShRNA-NC

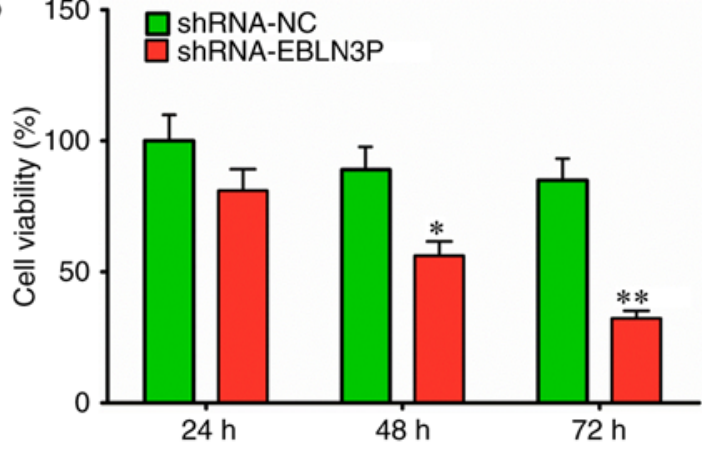

D

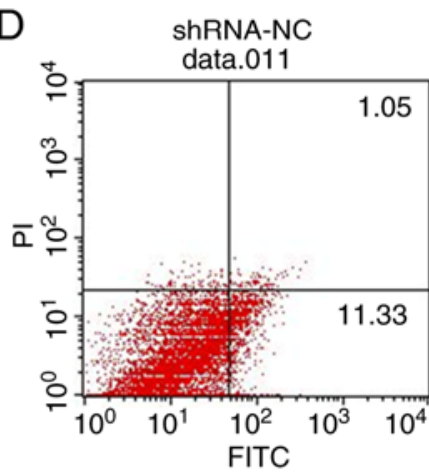

F

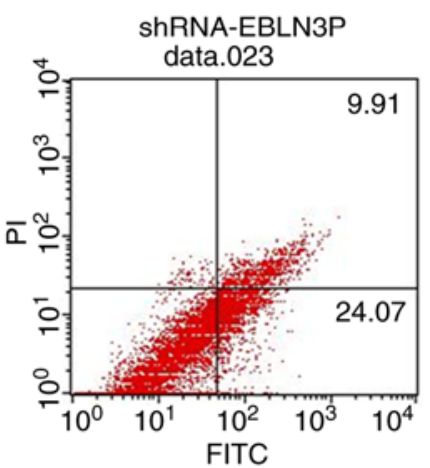

ShRNA/ ShRNA/

NC EBLN3P
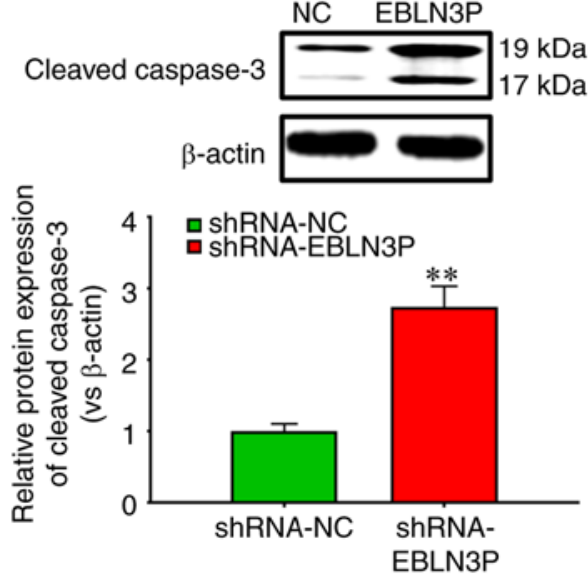

Figure 4. IncRNA EBLN3P promotes the viability and inhibits the apoptosis of normal SGNs. (A) Normal SGNs were treated with lncRNA EBLN3P shRNA and negative control shRNA via transfection with Lipofectamine 3000. SGN cell viability was determined by MTT assay. (B) Cell survival of SGNs was measured by Trypan blue staining in SGNs treated with lncRNA EBLN3P shRNA and negative control shRNA. (C) Necrosis of SGNs was determined by detection of lactate dehydrogenase release to the medium in SGNs treated with lncRNA EBLN3P shRNA and negative control shRNA. (D) Flow cytometry was performed to observe the effect of lncRNA EBLN3P knockdown on the apoptosis of normal SGNs cells. (E and F) Western blot analysis and quantitative analysis were performed to observe the effect of lncRNA EBLN3P knockdown on the levels of cleaved caspase-3 in normal SGNs. "P<0.05 and ${ }^{* *} \mathrm{P}<0.01$ (Student's t-test) vs. negative controls. lncRNA, long non-coding RNA; SGN, spiral ganglion neuron; shRNA, small hairpin RNA.

anti-AGO2 RIP experiment was then performed using the SGNs by transiently overexpressing miR-204-5p. As shown in Fig. 2F, endogenous IncRNA EBLN3P pull-down by AGO2 was specifically enriched in the miR-204-5p-transfected SGNs, indicating that miR-204-5p is a bona fide lncRNA EBLN3P targeting miRNA. This result demonstrated that miR-204-5p bound to lncRNA EBLN3P without affecting the degradation of lncRNA EBLN3P. In the presence of AGO2 protein, IncRNA EBLN3P maintained a stable and strong interaction with miR-204-5p (Fig. 2B and C). However, in the absence of AGO2 protein, a negative association was observed between IncRNA EBLN3P and miR-204-5p (Fig. 2G-I).
IncRNA EBLN3P regulates the expression of TMPRSS3 through miR-204-5p in normal SGNs. Due to the complex associations between lncRNA EBLN3P, miR-204-5p and TMPRSS3, the regulatory effects of IncRNA EBLN3P on miR-204-5p and TMPRSS were further evaluated. The results revealed that IncRNA EBLN3P knockdown successfully down-regulated the expression of IncRNA EBLN3P (Fig. 3A), and decreased the activities of the TMPRSS3 3'-UTR reporter (Fig. 3B). Furthermore, IncRNA EBLN3P knockdown significantly downregulated the mRNA and protein expression of TMPRSS3 (Fig. 3C and D). In addition, the results demonstrated that overexpression of full-length 
A

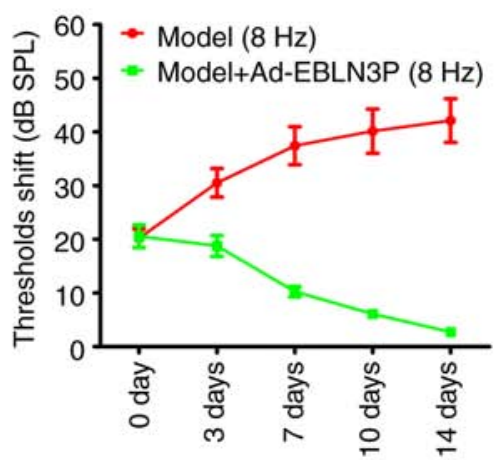

C

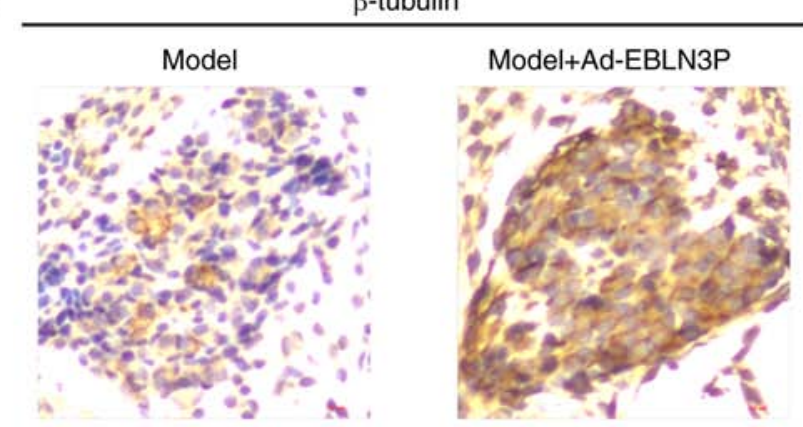

B

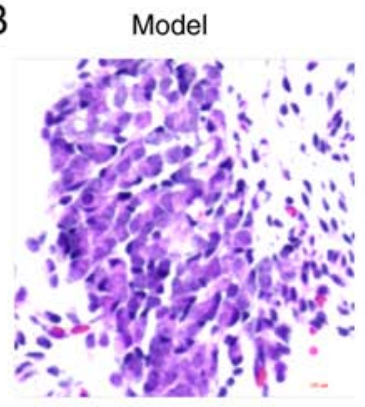

Model+Ad-EBLN3P

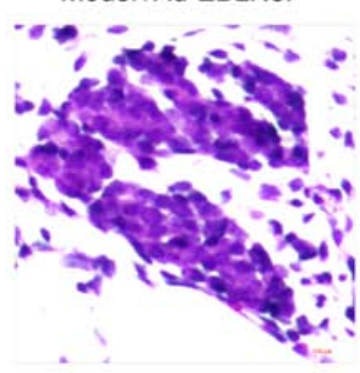

Figure 5. Recovery-promoting effect of Ad-EBLN3P on the structure and function of impaired organ of Corti and spiral ganglion. (A) Auditory brainstem response test was performed to evaluate the function of the organ of Corti and spiral ganglion in deafness models. Colored dots show the mean of the threshold shift to the $8-\mathrm{kHz}$ stimuli before and after injection of Ad-EBLN3P in deafness models. (B) Hematoxylin and eosin staining was applied to observe the recovering effect of long non-coding RNA EBLN3P on the structure of impaired organ of Corti and spiral ganglions. (C and D) Immunohistochemistry and quantitative analysis were performed to observe the levels of $\beta$-tubulin in cochlear spiral ganglion neurons. $\beta$-tubulin was used as an early marker for neurons. ${ }^{* *} \mathrm{P}<0.01$ (Student's t-test) vs. model.

lncRNA EBLN3P significantly upregulated the expression of lncRNA EBLN3P (Fig. 3E), and markedly elevated the activity of the TMPRSS3 3'-UTR reporter (Fig. 3F). However, when the SGNs were preliminarily treated with the inhibitor of miR-204-5p (anti-miR-204-5p), the overexpression of the full-length lncRNA EBLN3P had no obvious effect on the activity of the TMPRSS3 3'-UTR reporter (Fig. 3F). Similarly, the overexpression of full-length lncRNA EBLN3P markedly elevated the mRNA and protein expression levels of TMPRSS3. However, when the SGNs were preliminarily treated with an inhibitor of miR-204-5p, the overexpression of full-length lncRNA EBLN3P had no obvious effects on the mRNA or protein expression of TMPRSS3 (Fig. 3G-I).

lncRNA EBLN3P knockdown decreases the viability and promotes the apoptosis of normal SGNs. To determine the effects of lncRNA EBLN3P on the viability of normal SGNs, the targeted knockdown of IncRNA EBLN3P by shRNA was performed. The efficiency of plasmid transfection in neuronal cells was $20-25 \%$, as shown by fluorescence microscopy observation (data not shown). The positively transfected cells were further screened by flow cytometry following transfection with a GFP-labeled plasmid for $48 \mathrm{~h}$, prior to being subjected to subsequent experiments. Transfection with lncRNA EBLN3P shRNA markedly suppressed the viability of the SGNs at 48 and $72 \mathrm{~h}$, in contrast to transfection with negative control shRNA $(\mathrm{P}<0.05)$ (Fig. 4A). Trypan blue staining of the SGNs also revealed a significantly lower viability of the SGNs transfected with lncRNA EBLN3P shRNA relative to that of the control SGNs (Fig. 4B), which was confirmed by a significantly higher LDH release from the cultures of lncRNA EBLN3P shRNA-treated SGNs, indicating increased necrosis (Fig. 4C). Subsequently, the effect of lncRNA EBLN3P on the apoptosis of SGNs was further investigated. Transfection of the SGNs with lncRNA EBLN3P shRNA markedly promoted the early and late apoptosis of the SGNs compared with those transfected with shRNA NC $(\mathrm{P}<0.05)$ (Fig. 4D and E). Furthermore, the expression of caspase-3 was detected, which is closely associated with cell apoptosis. Transfection of the normal SGNs with lncRNA EBLN3P shRNA markedly promoted the expression of cleaved caspase-3 in SGNs, in comparison with the SGNs transfected with shRNA NC (Fig. 4F). Thus, these results demonstrated that the knockdown of lncRNA EBLN3P contributed to the reduced survival of normal SGNs and to the increased apoptosis of normal SGNs.

Recovery-promoting effect of IncRNA EBLN3P on the structure and function of impaired organs of Corti and SGNs in a model of deafness. Due to the positive regulatory effect of lncRNA EBLN3P on the survival of SGNs, the healing effect of IncRNA EBLN3P in models of deafness was then investigated. Adenovirus comprising overexpression vectors of lncRNA EBLN3P was injected into the mice in the models of deafness. The results of the ARB test revealed that compared with the model group, Ad-EBLN3P significantly decreased the threshold (Fig. 5A). $\mathrm{H} \& \mathrm{E}$ staining demonstrated that Ad-EBLN3P promoted the recovery of the structure of the impaired organ of Corti and SGNs (Fig. 5B). Subsequently, by detecting the expression of $\beta$-tubulin in cochlear SGNs, it was observed that Ad-EBLN3P increased the viability of neurons in vivo (Fig. $5 \mathrm{C}$ and $\mathrm{D}$ ). 


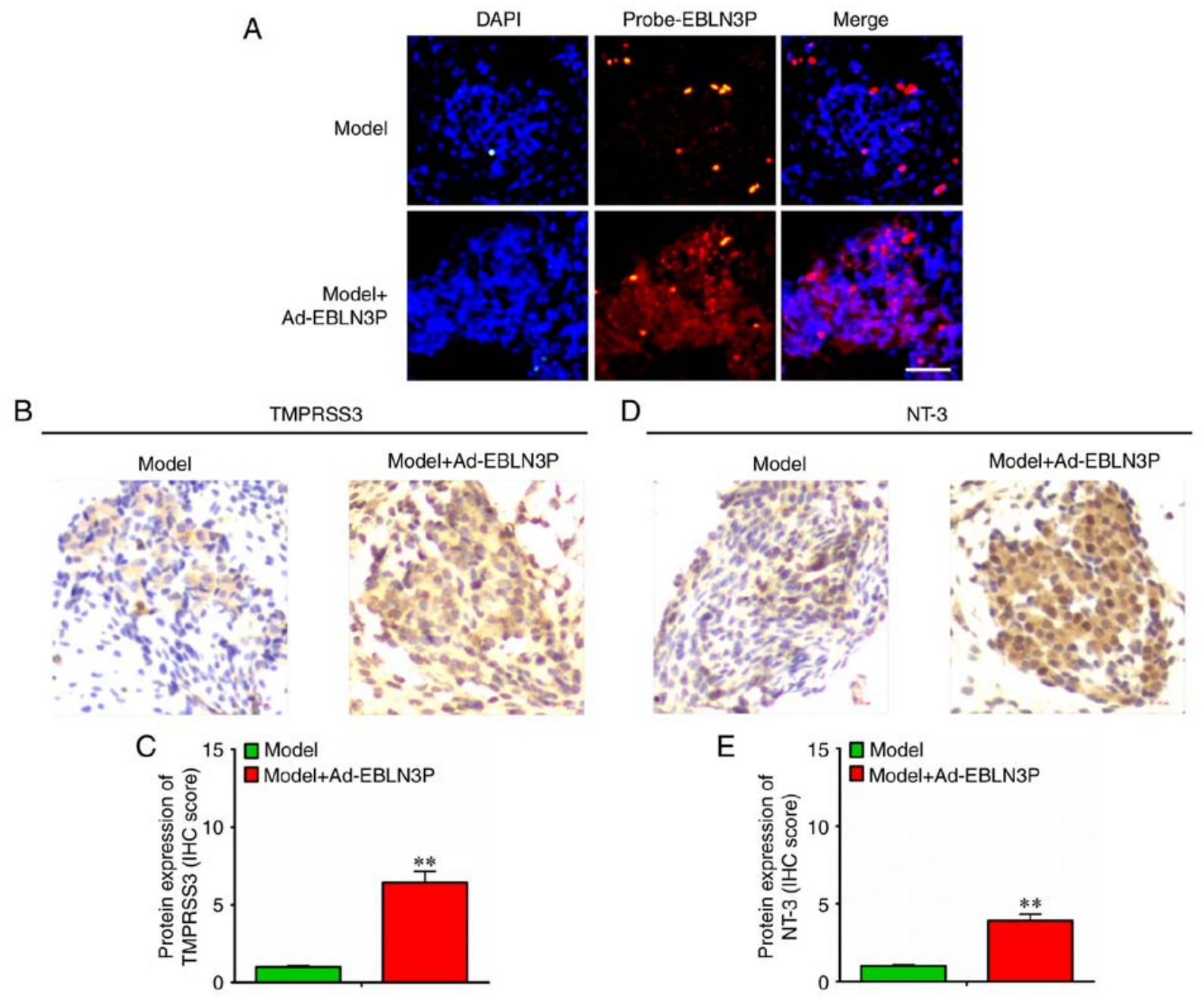

Figure 6. Expression of 1ncRNA EBLN3P, TMPRSS3 and NT-3 in the cochlear SGNs of deafness models. (A) Fluorescence in situ hybridization was performed to observe the expression of lncRNA EBLN3P in the cochlear SGNs of deafness models. (B and C) IHC and quantitative analysis were performed to observe the expression of TMPRSS3 in the cochlear SGNs of deafness models. (D and E) IHC and quantitative analysis were performed to observe the expression of NT-3, an important growth factor that helps stimulate and control neurogenesis, in the cochlear SGNs models of deafness. ${ }^{* *} \mathrm{P}<0.01$ (Student's t-test) vs. model. lncRNA, long non-coding RNA; SGN, spiral ganglion neuron; TMPRSS3, transmembrane protease, serine 3; NT-3, neurotrophin-3; IHC, immunohistochemistry.



Figure 7. Schematic model of lncRNA EBLN3P functioning as a competing endogenous RNA for miR-204-5p and regulating the expression of TMPRSS3. miR-204-5p could bind to the 3'-untranslated region of TMPRSS3 to inhibit the expression of TMPRSS3. IncRNA EBLN3P could bind to miR-204-5p at two sites, '314-322' and '973-981', in the 1,200-nt gene fragment at the 5'-end of EBLN3P, and block the regulatory function of miR-204-5p on TMPRSS3 expression. lncRNA, long non-coding RNA; miR, microRNA; TMPRSS3, transmembrane protease, serine 3. 
In subsequent experiments, the expression of Ad-EBLN3P in cochlear SGNs was detected, and it was observed that lncRNA EBLN3P and TMPRSS3 protein were overexpressed in the neurons (Fig. 6A-C). Furthermore, NT-3 was also overexpressed in the neurons (Fig. 6D and E).

All the above-mentioned results indicated that IncRNA EBLN3P promoted the recovery of the function of impaired SGNs by competitively binding to miR-204-5p and regulating the targeted gene TMPRSS3 expression (Fig. 7).

\section{Discussion}

Hearing loss is a frequent disorder and a highly heterogeneous disease, with an incidence rate of 1/1,000 individuals worldwide. Multiple genetic and environmental causes can lead to hearing loss, and SNHL is among the most common ones. Several studies have reported the degeneration of SGNs and/or sensory hair cells in SNHL $(1,37,38)$. Moreover, SGNs are poorly regenerated in the mammalian inner ear (39). Therefore, numerous studies have focused on the prevention of SGN progressive degeneration. Recently, numerous molecular genetic studies have been conducted on the role of the TMPRSS3 gene in autosomal recessive non-syndromic hearing loss (40-42). Currently, 16 different TMPRSS3 gene mutations have been identified to play key roles in the development of the inner ear (40-45). Lee et al reported TMPRSS3 gene mutations in populations of East Asia (46). Previous studies have demonstrated that kanamycin injection can lead to a reduction in TMPRSS3 expression in the cochlea, implying vital roles of the TMPRSS3 gene in normal cochlea function (47). A previous by the authors also suggested a high expression of the TMPRSS3 gene in SGNs of mouse cochleae (13). It has also been demonstrated that miR-204-5p suppresses the survival of cochlear SGNs in vitro by targeting the TMPRSS3 gene (14). However, the upstream regulatory mechanism of miR-204-5p has not been fully elucidated to date.

lncRNA EBLN3P was discovered and characterized during the Human Genome Project in 2002 (48), and was confirmed in another genome characterization in 2004 (49). Previous studies on the genomic characterization of functional lncRNA loci in human cells also found the existence of lncRNA EBLN3P (50). However, no functional or mechanistic reports have been published thus far, at least to the best of our knowledge. Some studies have suggested the potential role of lncRNAs as ceRNAs (51-53); however, the mechanism of the discovered lncRNAs was not well defined.

The present study firstly demonstrated that lncRNA EBLN3P functions as a ceRNA by regulating miR-204-5p in normal SGNs. In vitro functional experiments demonstrated that lncRNA EBLN3P promoted the recovery of the viability of normal SGNs and inhibited the apoptosis of normal SGNs. Moreover, the recovery-promoting effect of lncRNA EBLN3P on the structure and function of impaired SGNs from the mice in the models of deafness was demonstrated, and its molecular mechanisms may involve binding to miR-204-5p and the regulation of the expression of its targeted gene TMPRSS3. NT-3 is one of the important growth factors that helps to stimulate and control neurogenesis. Its overexpression in neurons also confirmed the recovery-promoting function of lncRNA EBLN3P on the structure and function of impaired SGNs in deafness models.
In conclusion, the findings of the present study demonstrated that lncRNA EBLN3P promoted the recovery of the function of impaired SGNs by competitively binding to miR-204-5p and regulating TMPRSS3 expression, suggesting that IncRNA EBLN3P may be a potential therapeutic target in SNHL.

\section{Acknowledgements}

Not applicable.

\section{Funding}

This study was supported by the National Natural Science Foundation of China (grant no. 81570928).

\section{Availability of data and materials}

All the data generated or analyzed during this study are included in this published article or are available from the corresponding author on reasonable request.

\section{Authors' contributions}

WJ, AP and ZZ designed the study. WJ, AP and ZZ also conducted the reviewing and editing of the manuscript. WJ, $\mathrm{AP}, \mathrm{YC}$ and BP performed the experiments and data analysis. All authors had full access to all the data in the study and take responsibility for the integrity of the data and the accuracy of the data analysis. WJ and AP wrote the manuscript. All authors read and approved the final manuscript.

\section{Ethics approval and consent to participate}

All animal experiments were approved by Animal Care and Use Committee at Xiangya School of Medicine from Central South University.

\section{Patient consent for publication}

Not applicable.

\section{Competing interests}

The authors declare that they have no competing interests.

\section{References}

1. Charizopoulou N, Lelli A, Schraders M, Ray K, Hildebrand MS, Ramesh A, Srisailapathy CR, Oostrik J, Admiraal RJ, Neely HR, et al: Gipc3 mutations associated with audiogenic seizures and sensorineural hearing loss in mouse and human. Nat Commun 2: 201, 2011.

2. Clark GM: Cochlear Implants: Fundamentals and applications Graeme Clark. Springer, New York, 2003.

3. Feghali JG, Lefebvre PP, Staecker H, Kopke R, Frenz DA, Malgrange B, Liu W, Moonen G, Ruben RJ and Van de Water TR: Mammalian auditory hair cell regeneration/repair and protection: A review and future directions. Ear Nose Throat J 77: 276 , 280, 282-285, 1998.

4. Hardie NA and Shepherd RK: Sensorineural hearing loss during development: Morphological and physiological response of the cochlea and auditory brainstem. Hear Res 128: 147-165, 1999. 
5. Dodson HC and Mohuiddin A: Response of spiral ganglion neurones to cochlear hair cell destruction in the guinea pig. J Neurocytol 29: 525-537, 2000.

6. Dror AA and Avraham KB: Hearing loss: Mechanisms revealed by genetics and cell biology. Annu Rev Genet 43: 411-437, 2009.

7. Bindu LH and Reddy PP: Genetics of aminoglycoside-induced and prelingual non-syndromic mitochondrial hearing impairment: A review. Int J Audiol 47: 702-707, 2008.

8. Wallrapp C, Hähnel S, Müller-Pillasch F, Burghardt B, Iwamura T, Ruthenbürger M, Lerch MM, Adler G and Gress TM: A novel transmembrane serine protease (TMPRSS3) overexpressed in pancreatic cancer. Cancer Res 60: 2602-2606, 2000.

9. Bugge TH, Antalis TM and Wu Q: Type II transmembrane serine proteases. J Biol Chem 284: 23177-23181, 2009.

10. Guipponi M, Toh MY, Tan J, Park D, Hanson K, Ballana E, Kwong D, Cannon PZ, Wu Q, Gout A, et al: An integrated genetic and functional analysis of the role of type II transmembrane serine proteases (TMPRSSs) in hearing loss. Hum Mutat 29: 130-141, 2008

11. Guipponi M, Vuagniaux G, Wattenhofer M, Shibuya K, Vazquez M, Dougherty L, Scamuffa N, Guida E, Okui M, Rossier C, et al: The transmembrane serine protease (TMPRSS3) mutated in deafness DFNB8/10 activates the epithelial sodium channel $(\mathrm{ENaC})$ in vitro. Hum Mol Genet 11: 2829-2836, 2002.

12. Fasquelle L, Scott HS, Lenoir M, Wang J, Rebillard G, Gaboyard S, Venteo S, François F, Mausset-Bonnefont AL, Antonarakis SE, et al: Tmprss3, a transmembrane serine protease deficient in human DFNB8/10 deafness, is critical for cochlear hair cell survival at the onset of hearing. J Biol Chem 286: 17383-17397, 2001.

13. Ge S, Wang Q, Peng A, Wu W and Xie D: Expression of proteinase TMPRSS3 in mouse cochlea. Zhong Nan Da Xue Xue Bao Yi Xue Ban 36: 794-798, 2011 (In Chinese).

14. Li Y, Peng A, Ge S, Wang Q and Liu J: MiR-204 suppresses cochlear spiral ganglion neuron survival in vitro by targeting TMPRSS3. Hear Res 314: 60-64, 2014.

15. Ponting CP, Oliver PL and Reik W: Evolution and functions of long noncoding RNAs. Cell 136: 629-641, 2009.

16. Mercer TR, Dinger ME and Mattick JS: Long non-coding RNAs: Insights into functions. Nat Rev Genet 10: 155-159, 2009.

17. Wilusz JE, Sunwoo H and Spector DL: Long noncoding RNAs: Functional surprises from the RNA world. Genes Dev 23: 1494-1504, 2009.

18. Feng J, Bi C, Clark BS, Mady R, Shah P and Kohtz JD: The Evf-2 noncoding RNA is transcribed from the Dlx-5/6 ultraconserved region and functions as a Dlx-2 transcriptional coactivator. Genes Dev 20: 1470-1484, 2006.

19. Martianov I, Ramadass A, Serra Barros A, Chow N and Akoulitchev A: Repression of the human dihydrofolate reductase gene by a non-coding interfering transcript. Nature 445 : 666-670, 2007

20. Wang X, Arai S, Song X, Reichart D, Du K, Pascual G, Tempst P, Rosenfeld MG, Glass CK and Kurokawa R: Induced ncRNAs allosterically modify RNA-binding proteins in cis to inhibit transcription. Nature 454: 126-130, 2008.

21. Bächinger D, Horvath L,Eckhard A, Goosmann MM,Honegger T, Gassmann M, Vogel J and Naldi AM: Neuronal erythropoietin overexpression is protective against kanamycin-induced hearing loss in mice. Toxicol Lett 291: 121-128, 2018

22. Ishikura F, Takano Y and Ueyama T: Acute effects of beta-blocker with intrinsic sympathomimetic activity on stress-induced cardiac dysfunction in rats. J Cardiol 60: 470-474, 2012.

23. Lina IA and Lauer AM: Rapid measurement of auditory filter shape in mice using the auditory brainstem response and notched noise. Hear Res 298: 73-79, 2013.

24. Tang X Gao M, Feng S and Su J: Gamma-aminobutyric acid A receptor and $\mathrm{N}$-methyl-D-aspartate receptor subunit expression in rat spiral ganglion neurons. Neural Regen Res 5: 1-3, 2010.

25. Martinez-Monedero R, Corrales CE, Cuajungco MP, Heller S and Edge AS: Reinnervation of hair cells by auditory neurons after selective removal of spiral ganglion neurons. J Neurobiol 66 : 319-331, 2006.

26. Klahr AC, Dickson CT and Colbourne F: Seizure activity occurs in the collagenase but not the blood infusion model of striatal hemorrhagic stroke in rats. Transl Stroke Res 6: 29-38, 2015.

27. Livak KJ and Schmittgen TD: Analysis of relative gene expression data using real-time quantitative PCR and the 2(-Delta Delta C(T)) method. Methods 25: 402-408, 2001
28. Wang Y, Jiang SW, Liu X, Niu L, Ge XL, Zhang JC, Wang HR, Fei AH, Gao CJ and Pan SM: Degradation of TRPML1 in neurons reduces neuron survival in transient global cerebral ischemia. Oxid Med Cell Longev 2018: 4612727, 2018

29. Song L, Tang S, Dong L, Han X, Cong L, Dong J, Han X, Zhang Q, Wang Y and Du Y: The neuroprotection of KIBRA in promoting neuron survival and against amyloid $\beta$-induced apoptosis. Front Cell Neurosci 13: 137, 2019.

30. Bogetofte H, Jensen P, Ryding M, Schmidt SI, Okarmus J, Ritter L, Worm CS, Hohnholt MC, Azevedo C, Roybon L, et al: PARK2 mutation causes metabolic disturbances and impaired survival of human iPSC-derived neurons. Front Cell Neurosci 13: 297, 2019.

31. Voss U, Sand E, Hellström PM and Ekblad E: Glucagon-like peptides 1 and 2 and vasoactive intestinal peptide are neuroprotective on cultured and mast cell co-cultured rat myenteric neurons. BMC Gastroenterol 12: 30, 2012.

32. Ishimoto S, Kawamoto K, Kanzaki S and Raphael Y: Gene transfer into supporting cells of the organ of Corti. Hear Res 173: $187-197,2002$.

33. Chandradoss SD, Schirle NT, Szczepaniak M, MacRae IJ and Joo C: A dynamic search process underlies MicroRNA targeting. Cell 162: 96-107, 2015

34. Nishi K, Takahashi T, Suzawa M, Miyakawa T, Nagasawa T, Ming Y, Tanokura M and Ui-Tei K: Control of the localization and function of a miRNA silencing component TNRC6A by argonaute protein. Nucleic Acids Res 43: 9856-9873, 2015.

35. Schraivogel D, Schindler SG, Danner J, Kremmer E, Pfaff J, Hannus S, Depping R and Meister G: Importin- $\beta$ facilitates nuclear import of human $\mathrm{GW}$ proteins and balances cytoplasmic gene silencing protein levels. Nucleic Acids Res 43: 7447-7461, 2015.

36. Weinmann L, Höck J, Ivacevic T, Ohrt T, Mütze J, Schwille P, Kremmer E, Benes V, Urlaub H and Meister G: Importin 8 is a gene silencing factor that targets argonaute proteins to distinct mRNAs. Cell 136: 496-507, 2009.

37. Kim YR, Baek JI, Kim SH, Kim MA, Lee B, Ryu N, Kim KH, Choi DG, Kim HM, Murphy MP, et al: Therapeutic potential of the mitochondria-targeted antioxidant MitoQ in mitochondrial-ROS induced sensorineural hearing loss caused by Idh2 deficiency. Redox Biol 20: 544-555, 2019.

38. Hu H, Ye B, Zhang L, Wang Q, Liu Z, Ji S, Liu Q, Lv J, Ma Y, $\mathrm{Xu}$ Y, et al: Efr3a insufficiency attenuates the degeneration of spiral ganglion neurons after hair cell loss. Front Mol Neurosci 10: 86, 2017.

39. Zhang PZ, He Y, Jiang XW, Chen FQ, Chen Y, Shi L, Chen J, Chen X, Li X, Xue T, et al: Stem cell transplantation via the cochlear lateral wall for replacement of degenerated spiral ganglion neurons. Hear Res 298: 1-9, 2013.

40. Lee K, Khan S, Islam A, Ansar M, Andrade PB, Kim S, Santos-Cortez RL, Ahmad W and Leal SM: Novel TMPRSS3 variants in Pakistani families with autosomal recessive non-syndromic hearing impairment. Clin Genet 82: 56-63, 2012.

41. Walsh T, Abu Rayan A, Abu Sa'ed J, Shahin H, Shepshelovich J, Lee MK, Hirschberg K, Tekin M, Salhab W, Avraham KB, et al: Genomic analysis of a heterogeneous Mendelian phenotype: Multiple novel alleles for inherited hearing loss in the Palestinian population. Hum Genomics 2: 203-211, 2006

42. Wattenhofer M, Sahin-Calapoglu N, Andreasen D, Kalay E, Caylan R, Braillard B, Fowler-Jaeger N, Reymond A, Rossier BC, Karaguzel A and Antonarakis SE: A novel TMPRSS3 missense mutation in a DFNB8/10 family prevents proteolytic activation of the protein. Hum Genet 117: 528-535, 2005.

43. Charif M, Abidi O, Boulouiz R, Nahili H, Rouba H, Kandil M, Delprat B, Lenaers G and Barakat A: Molecular analysis of the TMPRSS3 gene in Moroccan families with non-syndromic hearing loss. Biochem Biophys Res Commun 419: 643-647, 2012.

44. Hutchin T, Coy NN, Conlon H, Telford E, Bromelow K, Blaydon D, Taylor G, Coghill E, Brown S, Trembath R, et al: Assessment of the genetic causes of recessive childhood non-syndromic deafness in the UK-implications for genetic testing. Clin Genet 68: 506-512, 2005.

45. Masmoudi S, Antonarakis SE, Schwede T, Ghorbel AM, Gratri M, Pappasavas MP, Drira M, Elgaied-Boulila A, Wattenhofer M, Rossier C, et al: Novel missense mutations of TMPRSS3 in two consanguineous Tunisian families with non-syndromic autosomal recessive deafness. Hum Mutat 18: $101-108,2001$ 
46. Lee J, Baek JI, Choi JY, Kim UK, Lee SH and Lee KY: Genetic analysis of TMPRSS3 gene in the Korean population with autosomal recessive nonsyndromic hearing loss. Gene 532: 276-280, 2013.

47. Eppsteiner RW, Shearer AE, Hildebrand MS, Deluca AP, Ji H Dunn CC, Black-Ziegelbein EA, Casavant TL, Braun TA, Scheetz TE, et al: Prediction of cochlear implant performance by genetic mutation: The spiral ganglion hypothesis. Hear Res 292: 51-58, 2012.

48. Strausberg RL, Feingold EA, Grouse LH, Derge JG, Klausner RD, Collins FS, Wagner L, Shenmen CM, Schuler GD, Altschul SF, et al: Generation and initial analysis of more than 15,000 full-length human and mouse cDNA sequences. Proc Natl Acad Sci USA 99: 16899-16903, 2002.

49. Ota T, Suzuki Y, Nishikawa T, Otsuki T, Sugiyama T, Irie R, Wakamatsu A, Hayashi K, Sato H, Nagai K, et al: Complete sequencing and characterization of 21,243 full-length human cDNAs. Nat Genet 36: 40-45, 2004

50. Liu SJ, Horlbeck MA, Cho SW, Birk HS, Malatesta M, He D, Attenello FJ, Villalta JE, Cho MY, Chen Y, et al: CRISPRi-based genome-scale identification of functional long noncoding RNA loci in human cells. Science 355: pii: aah7111, 2017.
51. Liang H, Pan Z, Zhao X, Liu L, Sun J, Su X, Xu C, Zhou Y, Zhao D, Xu B, et al: LncRNA PFL contributes to cardiac fibrosis by acting as a competing endogenous RNA of let-7d. Theranostics 8: 1180-1194, 2018.

52. Qiu YY, Wu Y, Lin MJ, Bian T, Xiao YL and Qin C: LncRNA-MEG3 functions as a competing endogenous RNA to regulate Treg/Th17 balance in patients with asthma by targeting microRNA-17/ ROR $\gamma$ t. Biomed Pharmacother 111: 386-394, 2019.

53. Ji Q, Cai G, Liu X, Zhang Y, Wang Y, Zhou L, Sui H and Li Q: MALAT1 regulates the transcriptional and translational levels of proto-oncogene RUNX2 in colorectal cancer metastasis. Cell Death Dis 10: 378, 2019.

This work is licensed under a Creative Commons Attribution-NonCommercial-NoDerivatives 4.0 International (CC BY-NC-ND 4.0) License. 